\title{
Antenna Selection for Energy-Efficient Dual-Functional Radar-Communication Systems
}

\author{
Iman Valiulahi, Christos Masouros, Senior Member, IEEE, Abdelhamid Salem, Member, IEEE, and Fan Liu, \\ Member, IEEE
}

\begin{abstract}
This paper studies antenna selection for an energyefficient millimeter-wave (mmWave) dual-functional radarcommunication (DFRC) system, communicating with multiple users while sensing a point target. To obtain a hardware efficient DFRC system, we propose an optimization problem that minimizes the Cramér-Rao bound $(\mathrm{CRB})$ for tracking the target while guaranteeing the communication quality of service $(\mathrm{QoS})$, and selects the best active antennas using $\ell_{0}$ norm. To address the non-convexity of the proposed problem, we relax the radar and communication constraints using semidefinite programming (SDP) and employ an $\ell_{1, \infty}$ norm, which promotes group sparsity, instead of the $\ell_{0}$ norm. Numerical results show that the proposed approach obtains better performance in terms of the CRB compared to the conventional approach while reducing the number of radio frequency (RF) chains and total power consumption at the transmitter with almost the same computational complexity.
\end{abstract}

Index Terms-Dual-functional radar-communication, antenna selection, precoding, compressive sensing.

\section{INTRODUCTION}

Millimeter wave (mmWave) dual-functional radarcommunication (DFRC) systems have attracted a substantial attention over the past few years owing to their ability to simultaneously track targets while serving communication links. They are known to provide a spectrum-, energy-, and hardware-efficient approach where a single transmission serves both sensing and communications [1].

Generally speaking, conventional approaches for the DFRC systems can be categorized in three ways: radar-centric, communication-centric, and joint radar-communication designs. The radar-centric approaches modulate communication symbols onto classical radar waveforms to convey information [2]. In [3], the authors incorporated communication data into sidelobes of multi-input multi-output (MIMO) beampatterns and used the main lobe to track the target. In the communication-centric methods, standard communication signals are exploited to track the radar targets. For example, in [4], the authors employed orthogonal frequency division multiplexing (OFDM) systems for the radar tasks. In the joint design techniques, which are more related to our work, radar and communication signals were designed from the start for both radar and communication operations [5]. Most relevant to

Iman Valiulahi, Christos Masouros, and Abdelhamid Salem are with the Department of Electronic and Electrical Engineering, University College London, London WC1E 7JE, U.K. (e-mails: i.valiulahi@ucl.ac.uk; c.masouros@ucl.ac.uk). Fan Liu is with the Department of Electrical and Electronic Engineering, Southern University of Science and Technology, Shenzhen 518055, China (e-mail: liuf6@sustech.edu.cn). our focus, the work in [6] presented a joint design where the radar performance is optimized in the form of the Cramér-Rao bound (CRB) subject to communication and transmit power constraints.

Employing multiple antennas increases diversity which benefits both sensing and communicating. At the same time, one desires to constraint the number of radio frequency (RF) chains [7] since RF components such as digital-to-analog converters (DAC)s and power amplifiers (PA)s are expensive and typically consume significant power [7]. Hence, to enhance the energy efficiency and reduce implementation cost, it is crucial to limit the number of RF chains. Thus, the literature is abundant with antenna selection approaches, most recently in the context of massive MIMO [8].

To the best of our knowledge, this paper is the first work in the literature that studies antenna selection for the DFRC systems. To do so, we propose an optimization problem that minimizes the CRB constrained on the quality of service (QoS) requested by communication users while limiting the total power consumption with the best active antennas using the $\ell_{0}$ norm. Indeed, the proposed optimization aims to reduce the power consumption at electronic instruments and allocate the saved power to the transmitting power budget. The resulting problem is not convex and to address this, we relax the $\ell_{0}$ norm using the $\ell_{1, \infty}$ norm and propose semidefinite programming (SDP) to tackle the non-convexity of the CRB and QoS constraints. The total computational complexity of the proposed approach is derived as a function of the numbers of communication users and transmit antennas. Our results show that our joint precoding and antenna selection approach obtains a favorable trade-off between performance and hardware efficiency. Moreover, we numerically show that the computational complexity of the proposed approach is comparable to the closest related benchmark [6].

Throughout the paper, vectors and matrices are denoted by bold lowercase and uppercase letters, respectively, i.e. $(\boldsymbol{x}, \boldsymbol{X})$. We denote the transpose, hermitian of a matrix using $(\cdot)^{T}$ and $(\cdot)^{H}$, respectively. $\operatorname{tr}(\cdot)$ shows the trace operation. The $i$-th element of vector $\boldsymbol{x}$ is given by $x_{i}$. The $i$-th column and $n_{1}, n_{2}$-th element of matrix $\boldsymbol{X}$ are denoted by $\boldsymbol{x}_{i}$ and $X\left(n_{1}, n_{2}\right)$, respectively. For any vector $\boldsymbol{x} \in \mathbb{C}^{N}$, we denote the $\ell_{0}$ norm of $\boldsymbol{x},\|\boldsymbol{x}\|_{0}=\sum_{i=1}^{N} 1_{\left\{x_{i} \neq 0\right\}}$. Finally, the $\ell_{2}$ norm of a vector is shown by $\|\cdot\|_{2}$.

\section{System Model And Problem Formulation}

As shown in Fig. 1, we consider a mmWave DFRC node equipped with $N_{t}$ transmit and $N_{r}$ receive antennas, commu- 


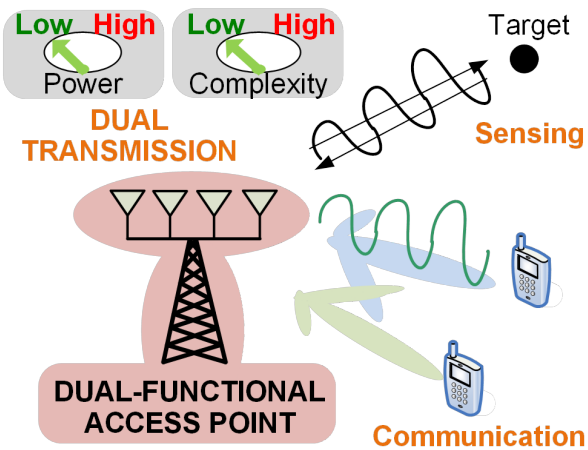

Fig. 1: The system model.

nicating with $K$ single-antenna users while tracking a point target. Without loss of generality, in this paper, it is assumed that $K<N_{t}<N_{r}$. Let us assume that $\boldsymbol{X} \in \mathbb{C}^{N_{t} \times L}$ as a dual-functional narrow-band transmit matrix where $L>N_{t}$ is the length of DFRC signal frames. Thus, the reflected matrix $\boldsymbol{Y}_{R} \in \mathbb{C}^{N_{r} \times L}$ from the target at the receiver antennas can be given by

$$
\boldsymbol{Y}_{R}=\boldsymbol{G} \boldsymbol{X}+\boldsymbol{N}_{R}
$$

where $\boldsymbol{G} \in \mathbb{C}^{N_{r} \times N_{t}}$ and $\boldsymbol{N}_{R} \in \mathbb{C}^{N_{r} \times L}$ are the target response matrix and additive white Gaussian noise (AWGN) matrix with variance $\sigma_{R}^{2}$ for each entry, respectively. By assuming the point target model, we can write $\boldsymbol{G}=\alpha \boldsymbol{b}(\theta) \boldsymbol{a}^{H}(\theta):=\alpha \boldsymbol{A}(\theta)$, where $\alpha$ is the complex reflection coefficient, which represents the effects of both the radar path-loss and cross-section of the target. The symbol $\theta$ shows the relative azimuth angle between the target and BS. Moreover, $\boldsymbol{a}(\theta) \in \mathbb{C}^{N_{t}}$ and $\boldsymbol{b}(\theta) \in$ $\mathbb{C}^{N_{r}}$ are steering vectors of the transmit and receive antenna arrays, respectively. For ease of notation, we use $\boldsymbol{A}$ instead of $\boldsymbol{A}(\theta)$. In this paper, uniform linear arrays (ULA)s are used for both the transmit and receiver antennas. Thus, $\boldsymbol{a}(\theta)=$ $\left[1, e^{j \frac{2 \pi}{\lambda} d \sin (\theta)} \cdots, e^{j \frac{2 \pi}{\lambda} d\left(N_{t}-1\right) \sin (\theta)}\right]^{T}$, where $d$ and $\lambda$ are the antenna spacing and the wavelength of the signal. Without loss of generality, we assume that $d=\frac{\lambda}{2}$. Similarly, we can obtain $\boldsymbol{b}(\theta)$ by considering $N_{r}$. The CRB for $\theta$ can be given by 2 , in the top of the next page [9] where $\boldsymbol{R}_{\boldsymbol{X}}=\frac{1}{L} \boldsymbol{X} \boldsymbol{X}^{H}$ and $\dot{\boldsymbol{A}}=\frac{d \boldsymbol{A}}{d \theta}$.

For the communication users, the received matrix $\boldsymbol{Y}_{C} \in$ $\mathbb{C}^{K \times L}$ can be written as

$$
\boldsymbol{Y}_{C}=\boldsymbol{H} \boldsymbol{X}+\boldsymbol{N}_{C}
$$

where $\boldsymbol{N}_{C} \in \mathbb{C}^{K \times L}$ is the AWGN matrix with variance $\sigma_{C}^{2}$ for each entry. Also, matrix $\boldsymbol{H}=\left[\boldsymbol{h}_{1}, \cdots, \boldsymbol{h}_{K}\right]^{H} \in \mathbb{C}^{K \times N_{t}}$ denotes the communication channel matrix in which $\boldsymbol{h}_{i} \in \mathbb{C}^{N_{t}}$ for $i \in\{1, \cdots, K\}$ represents the channel vector between the $i$-th user and the BS, which is assumed to be known at the BS. Here, let us write $\boldsymbol{X}$ as $\boldsymbol{X}=\boldsymbol{W} \boldsymbol{S}$, where $\boldsymbol{W}=\left[\boldsymbol{w}_{1}, \cdots, \boldsymbol{w}_{K}\right] \in \mathbb{C}^{N_{t} \times K}$ denotes the beamforming matrix in which $\boldsymbol{w}_{i} \in \mathbb{C}^{N_{t}}$ for $i \in\{1, \cdots, K\}$ is constructed by the beamforming weights of $N_{t}$ transmit antenna elements applied to the $i$-th communication user. Moreover, $\boldsymbol{S} \in \mathbb{C}^{K \times L}$ is the information-bearing matrix intended for $K$ users. Also, we assume that data streams are unite-power and orthogonal to each other, i.e., $\mathbb{E}\left[\boldsymbol{s}_{l} \boldsymbol{s}_{l}^{H}\right]=\frac{1}{L} \sum_{l=1}^{L} \boldsymbol{s}_{l} \boldsymbol{s}_{l}^{H}=\boldsymbol{I}$, where $s_{l}$ is the $l$-th column of $S$ and $\mathbb{E}$ denotes the ensemble average in which $\boldsymbol{I}$ is a $K \times K$ identity matrix. Moreover, $\boldsymbol{R}_{\boldsymbol{X}}=\sum_{k=1}^{K} \boldsymbol{w}_{k} \boldsymbol{w}_{k}^{H}$. Consequently, the total transmit power and signal-to-interference-plus-noise ratio (SINR) at the $k$-th user can be given by $P=\sum_{k=1}^{K}\left\|\boldsymbol{w}_{k}\right\|_{2}^{2}, \gamma_{k}=$ $\frac{\left|\boldsymbol{h}_{k}^{H} \boldsymbol{w}_{k}\right|^{2}}{\sum_{i=1, i \neq k}^{K}\left|\boldsymbol{h}_{k} \boldsymbol{w}_{i}\right|^{2}+\sigma_{C}^{2}}$, respectively. For our energy-efficient design, we employ the power consumption model of [10], where the total power consumption at the $\mathrm{BS}$ is given as $P_{t o t}=$ $P_{P A}+N_{t}\left(2 P_{D A C}+P_{R F}\right)$, where $P_{R F}=2 P_{M}+2 P_{L F}+P_{H B}$ in which $P_{M}, P_{L F}$, and $P_{H B}$ are the power of mixer, lowpass filter, and hybrid with buffer, respectively. The power of each DAC can be written as $P_{D A C}=c_{1} f q+c_{2} 2^{q}$ where $q$ is the resolution of DACs, $c_{2}$ is the coefficient of dynamic power consumption, $f$ is the sampling rate, and $c_{1}$ is the coefficient of static power consumption. $P_{P A}=\frac{P}{\eta}$ is the power of the PAs where $\eta$ denotes the PA efficiency and $P=\sum_{k=1}^{K}\left\|\boldsymbol{w}_{k}\right\|_{2}^{2}$ is the output of PAs.

Our target is to minimize the CRB and find the best active antennas and their corresponding beamforming vectors to reduce hardware components while guaranteeing communication services. To do so, we first introduce $\overline{\boldsymbol{w}}_{n}=$ $\left[w_{1}(n), \cdots, w_{K}(n)\right]^{T}$ as a vector that includes all the user weights applied to the $n$-th antenna. Then, we use $\tilde{\boldsymbol{w}}=$ $\left[\left\|\overline{\boldsymbol{w}}_{1}\right\|_{2}, \cdots,\left\|\overline{\boldsymbol{w}}_{N_{t}}\right\|_{2}\right]^{T}$ which consists the power of all $\overline{\boldsymbol{w}}_{n}$, for $n \in\left\{1, \cdots, N_{t}\right\}$. To exclude the $n$-th antenna from the transmission, all the elements of $\overline{\boldsymbol{w}}_{n}$ must be zero. The optimization problem to minimize the CRB constrained on communication performance and the total power consumption at the BS can be written as

$$
\begin{aligned}
\min _{\substack{\boldsymbol{w}_{i}, \forall i \in\{1, \cdots, K\}}} & \operatorname{CRB}(\theta) \\
& \text { s.t. } \gamma_{k} \geq \Gamma_{k}, \forall k, \\
& P_{P A}+P_{A}\|\tilde{\boldsymbol{w}}\|_{0} \leq P_{\text {tot }},
\end{aligned}
$$

where constraint (4a) stands for the QoS requested by the users in which $\Gamma_{k}$ is the SINR threshold. Constraint (4b) guarantees that the total power consumption is less than the total power budget in which $P_{A}=2 P_{D A C}+P_{R F}$ is the consumed power per antenna. Moreover, we know that $\|\tilde{\boldsymbol{w}}\|_{0} \leq N_{t}$. Since the beamforming and antenna selection are highly coupled, we consider both in constraint (4b). Solving problem (4) is challenging since all the constraints and objective value are non-convex. Constraint (4a) and the objective value are fractional. Constraint (4b) needs an exhaustive combinatorial sparsity search, hence is NP-hard and non-convex. Though $\operatorname{CRB}(\theta)$ is a function of $\theta$, the problem can be optimized with respect to $\boldsymbol{W}$. This means that the BS optimizes beampatterns towards an estimated direction, which can be used to track the location of a moving target [5], [6]. To tackle the above difficulties, we provide an efficient solution for the proposed problem in the next section.

\section{JOINT DFRC BEAMFORMING AND ANTENNA SELECTION}

Problem (4) is hard to solve because of non-convex constraints. However, in this section, we relax all non-convexity 


$$
\mathrm{CRB}(\theta)=\frac{\sigma_{R}^{2} \operatorname{tr}\left(\boldsymbol{A}^{H} \boldsymbol{A} \boldsymbol{R}_{\boldsymbol{X}}\right)}{2|\alpha|^{2} L\left(\operatorname{tr}\left(\dot{\boldsymbol{A}}^{H} \dot{\boldsymbol{A}} \boldsymbol{R}_{\boldsymbol{X}}\right) \operatorname{tr}\left(\boldsymbol{A}^{H} \boldsymbol{A} \boldsymbol{R}_{\boldsymbol{X}}\right)-\left|\operatorname{tr}\left(\dot{\boldsymbol{A}}^{H} \boldsymbol{A} \boldsymbol{R}_{\boldsymbol{X}}\right)\right|^{2}\right)},
$$

issues one by one. One possible solution for relaxing the non-convexity of the $\ell_{0}$ norm might be employing the $\ell_{1}$ norm which is its closest convex approximation. However, applying the $\ell_{1}$ norm is not single-handedly sufficient for antenna selection. Indeed, the $\ell_{1}$ norm obtains a sparse solution per transmission, $\boldsymbol{w}_{k}$. However, the zero elements do not essentially align to the same antenna. Thus, a regularization norm that can simultaneously promote the sparsity of all the entries of $\overline{\boldsymbol{w}}_{n}$ is needed. To do so, we use the square of $\ell_{1, \infty}$ norm, $\|\boldsymbol{w}\|_{1, \infty}=\sum_{n=1}^{N_{t}} \max _{k=1, \cdots, K}\left|w_{k}(n)\right|$, in which $\boldsymbol{w}=\left[\boldsymbol{w}_{1}^{T}, \cdots, \boldsymbol{w}_{K}^{T}\right]^{T}$, to relax the non-convex issues of the $\ell_{0}$ norm. It is worth noting that $\|\cdot\|_{1, \infty}$ is a group-sparsity norm, which is a combination of $\ell_{1}$ and infinity norms. Thus, it can align the non-zero elements of each $\boldsymbol{w}_{k}$ to the same antenna. Consequently, the optimization problem can be recast as

$$
\begin{aligned}
\min _{\substack{\boldsymbol{w}_{i}, \forall i \in\{1, \cdots, K\}}} & \operatorname{CRB}(\theta) \\
& \text { s.t. } \gamma_{k} \geq \Gamma_{k}, \forall k, \\
& \frac{\sum_{k=1}^{K}\left\|\boldsymbol{w}_{k}\right\|_{2}^{2}}{\eta}+P_{A}\|\boldsymbol{w}\|_{1, \infty}^{2} \leq P_{t o t} .
\end{aligned}
$$

The square of $\ell_{1, \infty}$ can be written as

$$
\begin{aligned}
& \left(\sum_{n=1}^{N_{t}} \max _{k=\{1, \cdots, K\}}\left|w_{k}(n)\right|\right)^{2} \\
& =\sum_{n_{1}=1}^{N_{t}} \sum_{n_{2}=1}^{N_{t}}\left(\max _{k=\{1, \cdots, K\}}\left|w_{k}\left(n_{1}\right)\right| \max _{k=\{1, \cdots, K\}}\left|w_{k}\left(n_{2}\right)\right|\right) \\
& =\sum_{n_{1}=1}^{N_{t}} \sum_{n_{2}=1}^{N_{t}} \max _{i, j \in\{1, \cdots, K\}}\left|W_{i, j}\left(n_{1}, n_{2}\right)\right| .
\end{aligned}
$$

Then, by defining $\tilde{\boldsymbol{W}}=\max _{k \in\{1, \cdots, K\}} W_{k, k}$, we can write

$$
\sum_{n_{1}=1}^{N_{t}} \sum_{n_{2}}^{N_{t}} \max _{k \in\{1, \cdots, K\}}\left|W_{k, k}\left(n_{1}, n_{2}\right)\right|=\operatorname{tr}\left(\mathbf{1}_{N_{t}} \tilde{\boldsymbol{W}}\right) .
$$

The above problem is still hard to solve because of the QoS and radar constraints. However, we relax these issues using SDP, which can be efficiently implemented by the interior point methods. Let us first define $\boldsymbol{W}_{k}=$ $\boldsymbol{w}_{k} \boldsymbol{w}_{k}^{H}, \boldsymbol{Q}_{k}=\boldsymbol{h}_{k} \boldsymbol{h}_{k}^{H}$. Then, we can write $\left\|\boldsymbol{w}_{k}\right\|_{2}^{2}=$ $\operatorname{tr}\left(\boldsymbol{w}_{k} \boldsymbol{w}_{k}^{H}\right)=\operatorname{tr}\left(\boldsymbol{W}_{k}\right),\left|\boldsymbol{h}_{k}^{H} \boldsymbol{w}_{k}\right|^{2}=\operatorname{tr}\left(\boldsymbol{h}_{k}^{H} \boldsymbol{w}_{k} \boldsymbol{h}_{k}^{H} \boldsymbol{w}_{k}\right)=$ $\operatorname{tr}\left(\boldsymbol{h}_{k} \boldsymbol{h}_{k}^{H} \boldsymbol{w}_{k} \boldsymbol{w}_{k}^{H}\right)=\operatorname{tr}\left(\boldsymbol{Q}_{k} \boldsymbol{W}_{k}\right)$. Consequently, constraint 4a can be converted to

$$
\operatorname{tr}\left(\boldsymbol{Q}_{k} \boldsymbol{W}_{k}\right)-\Gamma_{k} \sum_{i=1, i \neq k}^{k} \operatorname{tr}\left(\boldsymbol{Q}_{k} \boldsymbol{W}_{i}\right) \geq \Gamma_{k} \sigma_{C}^{2}, \forall k .
$$

In addition, by using the Schur complement condition [11], we can use the SDP form of

$$
\operatorname{tr}\left(\dot{\boldsymbol{A}}^{H} \dot{\boldsymbol{A}} \boldsymbol{R}_{\boldsymbol{X}}\right)-t-\left|\operatorname{tr}\left(\dot{\boldsymbol{A}}^{H} \boldsymbol{A} \boldsymbol{R}_{\boldsymbol{X}}\right)\right|^{2} \operatorname{tr}^{-1}\left(\boldsymbol{A}^{H} \boldsymbol{A} \boldsymbol{R}_{\boldsymbol{X}}\right) \geq 0,
$$

where $t$ is a constant variable in $\operatorname{CRB}(\theta)$ as below

$$
\left[\begin{array}{cc}
\operatorname{tr}\left(\dot{\boldsymbol{A}}^{H} \dot{\boldsymbol{A}} \sum_{k=1}^{K} \boldsymbol{W}_{k}\right)-t & \operatorname{tr}\left(\dot{\boldsymbol{A}}^{H} \boldsymbol{A} \sum_{k=1}^{K} \boldsymbol{W}_{k}\right) \\
\operatorname{tr}\left(\boldsymbol{A}^{H} \dot{\boldsymbol{A}} \sum_{k=1}^{K} \boldsymbol{W}_{k}\right) & \operatorname{tr}\left(\boldsymbol{A}^{H} \boldsymbol{A} \sum_{k=1}^{K} \boldsymbol{W}_{k}\right)
\end{array}\right] \succeq 0 .
$$

Note that $\operatorname{rank}\left(\boldsymbol{W}_{k}\right)=1$ and $\boldsymbol{W}_{k} \succeq 0, \forall k$ are required for the desired solution. By dropping the rank constraints, which are not convex, problem (5) can be written in a convex form as

$$
\begin{aligned}
& \min _{\boldsymbol{W}_{k}, \forall k}-t \\
& {\left[\begin{array}{c}
\operatorname{tr}\left(\dot{\boldsymbol{A}}^{H} \dot{\boldsymbol{A}} \sum_{k=1}^{K} \boldsymbol{W}_{k}\right)-t \quad \operatorname{tr}\left(\dot{\boldsymbol{A}}^{H} \boldsymbol{A} \sum_{k=1}^{K} \boldsymbol{W}_{k}\right) \\
\operatorname{tr}\left(\boldsymbol{A}^{H} \dot{\boldsymbol{A}} \sum_{k=1}^{K} \boldsymbol{W}_{k}\right) \quad \operatorname{tr}\left(\boldsymbol{A}^{H} \boldsymbol{A} \sum_{k=1}^{K} \boldsymbol{W}_{k}\right)
\end{array}\right] \succeq 0,} \\
& \operatorname{tr}\left(\boldsymbol{Q}_{k} \boldsymbol{W}_{k}\right)-\Gamma_{k} \sum_{i=1, i \neq k}^{k} \operatorname{tr}\left(\boldsymbol{Q}_{k} \boldsymbol{W}_{i}\right) \geq \Gamma_{k} \sigma_{C}^{2}, \forall k \\
& \frac{\sum_{k=1}^{K} \operatorname{tr}\left(\boldsymbol{W}_{k}\right)}{\eta}+P_{A} \operatorname{tr}\left(\mathbf{1}_{N_{t}} \tilde{\boldsymbol{W}}\right) \leq P_{t o t} \\
& \boldsymbol{W}_{k} \succeq 0, \tilde{\boldsymbol{W}} \geq\left|\boldsymbol{W}_{k}\right|, \forall k .
\end{aligned}
$$

This problem can be efficiently solved in polynomial time using off-the-shelf convex solvers such as CVX [12].

Regarding the rank relaxation applied to problem (8), the solution, $\hat{\boldsymbol{W}}_{k}, \forall k$ might not consist of rank-one matrices. Thus, the optimal beamforming vectors might not be directly achieved. However, using a Gaussian randomization technique proposed in [13], one can construct candidate sets of beamforming vectors from $\hat{\boldsymbol{W}}_{k}, \forall k$ and select the ones that can be scaled to satisfy constraints $8 \mathrm{Ba}$ and $8 \mathrm{~b}$ ) with the minimum power. If the resulting beamforming vectors are all rank-one matrices, the corresponding principal components with suitable scales are the optimal solutions. Note that these scales can be obtained using linear programming proposed in [13]. After obtaining the beamforming vectors from the above procedure, the number of active antennas can be calculated by counting the number of non-zero elements of beamforming vectors, which we call $U$. It is worth mentioning that our proposed approach can be extended to the multiple targets scenario using the Fisher Information Matrix (FIM) proposed in [14] and the augmented beamforming method, which was first studied in [6], at the cost of more computational complexity and stronger co-channel interference at the communication side.

Problem (8) is convex and can be solved using the interior point method by CVX. The complexity of solving such a problem is $\mathcal{O}\left((E+F)^{1.5} E^{2}\right)$, where $E$ and $F$ are the numbers of variables and constraints, respectively [12]. Therefore, the total computational complexity of problem $\overline{8}$ can be approximated by

$$
\left.\mathcal{O}\left(N_{t} K^{2}+3 K+3\right)^{1.5}\left(N_{t} K^{2}+1\right)^{2}\right) \approx \mathcal{O}\left(K^{7} N_{t}^{3.5}\right),
$$


TABLE I: Parameters for the total power consumption.

\begin{tabular}{|l|c|}
\hline$P_{M}$, mixers & $0.3 \mathrm{~mW}$ \\
\hline$P_{L F}$, low-pass filters & $14 \mathrm{~mW}$ \\
\hline$P_{H B}$, hybrid with buffers & $3 \mathrm{~mW}$ \\
\hline$c_{1}$ & $9 \times 10^{-12}$ \\
\hline$f$, the sampling frequency & $25 \mathrm{GHz}$ \\
\hline$q$, the resolution of DACs & 8 \\
\hline$c_{2}$ & $1.5 \times 10^{-5}$ \\
\hline $\begin{array}{l}P_{t o t}, \text { the total power } \\
\text { consumption }\end{array}$ & $75.5 \mathrm{~W}$ \\
\hline$\eta$, the efficiency of PAs & 0.4 \\
\hline
\end{tabular}

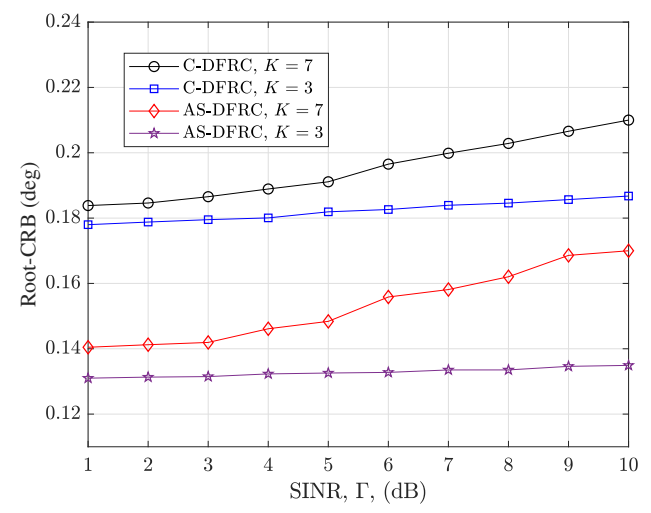

Fig. 2: A comparison between AS-DFRC and C-DFRC in terms of root-CRB for $N_{t}=16, N_{r}=20$ and the different number of users.

where shows that the effect of the number of users, $K$, is more than the number of antennas, $N_{t}$. From the computational complexity, we can understand that problem 8 can be implemented for the moderate number of communication users and transmit antennas in practice. We also show this by numerical simulations in the next section.

\section{Simulation Results}

This section provides Monte-Carlo simulations to evaluate the proposed antenna selection-based DFRC systems (AS-

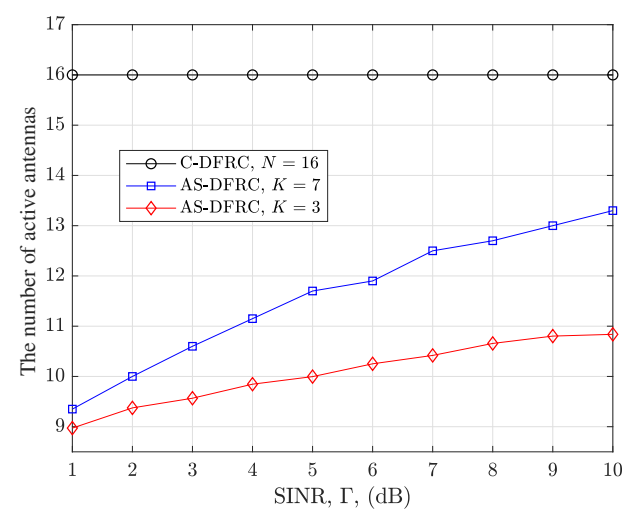

Fig. 3: A comparison between AS-DFRC and C-DFRC in terms of the number of active antennas versus the different SINR thresholds for $N_{t}=16, N_{r}=20$ and the different number of users.

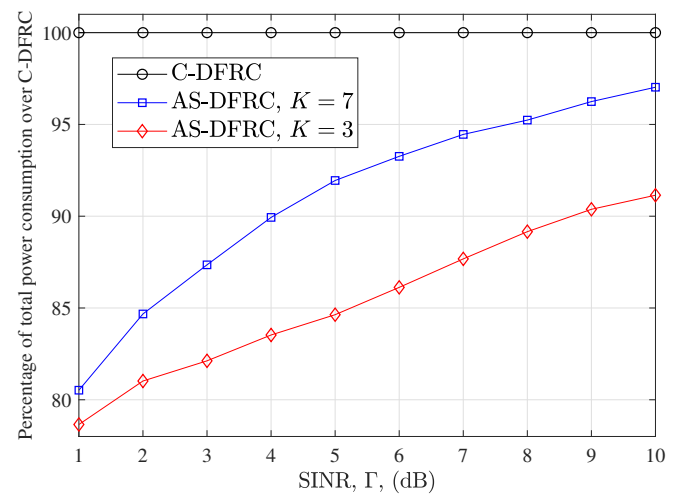

Fig. 4: The percentage of the C-DFRC power consumption at the BS versus the different values of SINR for AS-DFRC for $N_{t}=16, N_{r}=20$ and the different number of users.

DFRC). Without loss of generality, we assume that the target's angle is located at $\theta=0$ and the aim is to optimize the beamformers such that the CRB is minimized constrained on the communication QoS and number of active antennas. Indeed, we assume that an initial estimate of the target's angle is present at the DFRC node, which can be obtained using the super-resolution methods such as MUSIC [15] or the total variation norm minimization [16], then we aim to maximize the accuracy of the estimated angle by minimizing the CRB. Moreover, we set $L=N_{t}+1$, and $\sigma_{R}^{2}=\sigma_{C}^{2}=0.001$. We employ the technique in [6] as our benchmark, where the Cramér-Rao bound is minimized constrained on the communication performance and power budget. We call this approach "C-DFRC". The parameters for calculating the total power consumption at the BS are summarized in Table I.

We commence by evaluating the root-CRB in degree, $\sqrt{\mathrm{CRB}} * \frac{180}{\pi}$, of AS-DFRC and C-DFRC for the different number of users and SINR thresholds in Fig. 2. It is observed that AS-DFRC outperforms C-DFRC for both values of $K=3,7$. The root-CRB for both AS-DFRC and CDFRC increases by growing the SINR threshold, $\Gamma$, in decibel (dB). Moreover, the rate of this increase for $K=7$ is more than $K=3$ which is because of the larger value of the interference term. The proposed AS-DFRC reduces the power consumed by RF components and allocates the saved power to the transmit power budget. On the other hand, the dimension of $\boldsymbol{R}_{\boldsymbol{X}}$ in both the C-DFRC and AS-DFRC is the same as the proposed optimization optimally chooses $U$ antennas from the $N_{t}$ transmit antennas. Thus, as shown in Fig. 2, the proposed AS-DFRC obtains better performance in terms of the rootCRB than C-DFRC. To a fair comparison, we also investigate the number of active antennas and total power consumption at the BS below.

In Fig. 3, we plot the number of active antennas versus the SINR thresholds for the different values of $K$. It is observed that by increasing the value of $\Gamma$ or the number of communication users, more antennas must be employed to achieve the required QoS. In addition, the proposed AS-DFRC outperforms C-DFRC up to 7 and 5 antennas for the small and large values of SINR thresholds, respectively. Observing this 


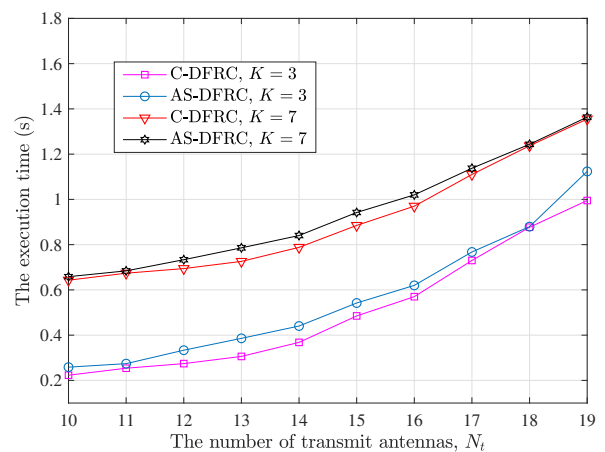

Fig. 5: The execution time versus the number of antennas for the proposed AS-DFRC and C-DFRC for the different values of $K$.

result together with Fig. 2, we can conclude that the AS-DFRC outperforms the C-DFRC in terms of the root-CRB with the less number of antennas and electronic equipment.

Note that in problem (8) we relaxed the non-convex antenna selection constraint using a group-sparsity norm, and then obtained $U$ from $\hat{\boldsymbol{W}}$. Regarding the fact that $\left.\mid \hat{\boldsymbol{W}}_{k}\right\rfloor<\tilde{\boldsymbol{W}}, \forall k$, we have $U \leq \operatorname{tr}\left(\mathbf{1}_{N_{t}} \tilde{\boldsymbol{W}}\right)$. While the constraint $8 \mathrm{cc}$ may be active, the true power consumption may be less than $P_{t o t}$. Accordingly, we plot the total power consumption of the proposed approach, $\hat{P}_{t o t}=\frac{\sum_{k=1}^{K} \operatorname{tr}(\hat{\boldsymbol{W}})}{\eta}+P_{A} U$, versus the SINR threshold in Fig. 4 to demonstrate the value of the proposed approach in reducing the total power consumption at the BS. More precisely, we used $P_{t o t}$ reported in Table 1 for C-DFRC and our proposed problem in 8 for the different values of SINR thresholds and number of communication users. It is observed from this figure that the percentage of total power consumption of AS-DFRC increases by growing the SINR threshold or number of communication users, however, it is upper bounded by the power consumed by C-DFRC. This is because of the fact that AS-DFRC decreases the power consumption at the BS by reducing the number of active antennas and their corresponding electronic devices and exploits the saved power for transmitting the signal, reducing the root-CRB compared to C-DFRC as shown in Fig. 4.

Finally, in Fig. 5, we compare the execution time of the AS-DFRC with C-DFRC in second (s) versus the number of transmit antennas for the different number of communication users. As shown by this figure, the implementation costs of both the approaches increase by growing the number of users or number of antennas. Moreover, the execution time of ASDFRC is almost the same with C-DFRC, which highlights the efficiency of the proposed approach in practical scenarios.

\section{CONClusion}

In this letter, we presented a non-convex problem to jointly optimize beamforming vectors and antenna selection in a mmWave DFRC node. The antenna selection, communication and radar performances are modeled by the $\ell_{0}$ norm, SINR threshold and CRB, respectively. We relaxed the $\ell_{0}$ constraint using the $\ell_{1, \infty}$ norm. In addition, we showed that the nonconvex radar and communication constraints can be relaxed by SDP. Simulation results showed a superior performancecomplexity trade-off for the proposed approach compared with [6]. An interesting future research direction is extending our proposed approach to the multiple targets scenario using the augmented beamforming matrix approach proposed in [6].

\section{ACKNOWLEDGMENT}

This project has received funding from the European Union's Horizon 2020 research and innovation program under the Marie Skłodowska-Curie grant agreement No 812991.

\section{REFERENCES}

[1] F. Liu, C. Masouros, A. Li, T. Ratnarajah, and J. Zhou, "Mimo radar and cellular coexistence: A power-efficient approach enabled by interference exploitation," IEEE Transactions on Signal Processing, vol. 66, no. 14, pp. 3681-3695, 2018.

[2] R. M. Mealey, "A method for calculating error probabilities in a radar communication system," IEEE Transactions on Space Electronics and Telemetry, vol. 9, no. 2, pp. 37-42, 1963.

[3] A. Hassanien, M. G. Amin, Y. D. Zhang, and F. Ahmad, "Dual-function radar-communications: Information embedding using sidelobe control and waveform diversity," IEEE Transactions on Signal Processing, vol. 64, no. 8, pp. 2168-2181, 2015.

[4] C. Sturm and W. Wiesbeck, "Waveform design and signal processing aspects for fusion of wireless communications and radar sensing," Proceedings of the IEEE, vol. 99, no. 7, pp. 1236-1259, 2011.

[5] F. Liu, C. Masouros, A. Li, H. Sun, and L. Hanzo, "MU-MIMO communications with mimo radar: From co-existence to joint transmission," IEEE Transactions on Wireless Communications, vol. 17, no. 4, pp. 2755-2770, 2018.

[6] F. Liu, Y.-F. Liu, A. Li, C. Masouros, and Y. C. Eldar, "Cramérrao bound optimization for joint radar-communication design," arXiv preprint arXiv:2101.12530, 2021.

[7] P. V. Amadori and C. Masouros, "Interference-driven antenna selection for massive multiuser mimo," Ieee transactions on vehicular technology, vol. 65, no. 8, pp. 5944-5958, 2015.

[8] A. García-Rodríguez, C. Masouros, and P. Rulikowski, "Reduced switching connectivity for power-efficient large scale antenna selection," IEEE Trans. Commun., DOI, vol. 10, 2016.

[9] Z. Ben-Haim and Y. C. Eldar, "On the constrained cramér-rao bound with a singular fisher information matrix," IEEE Signal Processing Letters, vol. 16, no. 6, pp. 453-456, 2009.

[10] L. N. Ribeiro, S. Schwarz, M. Rupp, and A. L. de Almeida, "Energy efficiency of mmwave massive mimo precoding with low-resolution dacs," IEEE Journal of Selected Topics in Signal Processing, vol. 12, no. 2, pp. 298-312, 2018.

[11] F. Zhang, The Schur complement and its applications, vol. 4. Springer Science \& Business Media, 2006.

[12] M. Grant and S. Boyd, "Cvx: Matlab software for disciplined convex programming, version 2.1," 2014.

[13] E. Karipidis, N. D. Sidiropoulos, and Z.-Q. Luo, "Quality of service and max-min fair transmit beamforming to multiple cochannel multicast groups," IEEE Transactions on Signal Processing, vol. 56, no. 3, pp. 1268-1279, 2008.

[14] J. Li, L. Xu, P. Stoica, K. W. Forsythe, and D. W. Bliss, "Range compression and waveform optimization for mimo radar: a cramér-rao bound based study," IEEE Transactions on Signal Processing, vol. 56, no. 1, pp. 218-232, 2007.

[15] J. Li and P. Stoica, "An adaptive filtering approach to spectral estimation and sar imaging," IEEE Transactions on Signal Processing, vol. 44, no. 6, pp. 1469-1484, 1996.

[16] I. Valiulahi, S. Daei, F. Haddadi, and F. Parvaresh, "Two-dimensional super-resolution via convex relaxation," IEEE Transactions on Signal Processing, vol. 67, no. 13, pp. 3372-3382, 2019. 